\title{
A PRESENÇA DA PRODUÇÃO CIENTÍFICA BRASILEIRA NA REVISTA KNOWLEDGE ORGANIZATION NO SÉCULO XXI
}

\author{
THE PRESENCE OF BRAZILIAN SCIENTIFIC \\ LITERATURE IN THE KNOWLEDGE ORGANIZATION \\ JOURNAL ON 21ST CENTURY
}

\author{
Manoela Ferreira da Silva ${ }^{a}$ \\ Isadora Victorino Evangelistab \\ José Augusto Chaves Guimarãesc
}

\begin{abstract}
RESUMO
Introdução: O periódico Knowledge Organization constitui hoje um dos principais veículos de comunicação científica no que diz respeito a questões de Organização do Conhecimento (OC). Objetivos: Dessa forma, considerou-se pertinente analisar como se apresenta o contexto brasileiro nessas comunicações, verificando quais são os autores e instituições mais produtivas, além de frequência de publicações, coautorias nacionais e colaborações com o exterior. Metodologia: Por meio da análise dos números publicados no período de 2001-2018, foi possível identificar uma forte presença de artigos de autoria brasileira, com um total de 51 produções. Resultados: Percebeu-se uma comunidade liderada pelos professores José Augusto Chaves Guimarães, Daniel Martínez-Ávila, Fábio de Assis Pinho e Renato Rocha de Souza, responsáveis por $28 \%$ de todas as publicações nesse período. Como instituições mais produtivas, destacam-se a Universidade Estadual Paulista - UNESP e a Universidade Federal de Minas Gerais - UFMG, instituições essas que possuíam ao menos um autor afiliado em metade das incidências institucionais. Percebe-se ainda, um forte crescimento no número de artigos nos anos mais recentes, em especial no ano de 2012 e 2014 em diante, responsáveis por quase 85\% do total de publicações brasileiras na revista. Em relação às coautorias nacionais, pode-se perceber um forte grupo preocupado com aspectos éticos e sociais da organização do conhecimento, estudos em linguagens documentárias, aspectos relativos à recuperação informacional, ontologias, web semântica, dentre outros. A respeito das coautorias

a Graduanda em Biblioteconomia pela Universidade Estadual Paulista "Júlio de Mesquita Filho" (UNESP). E-mail: manoelaf48@gmail.com

b Doutoranda em Ciência da Informação pelo Programa de Pós-Graduação da Universidade Estadual Paulista (UNESP). Bolsista da Fundação de Amparo à Pesquisa do Estado de São Paulo. E-mail: isadora.evangelista@unesp.br

c Doutor em Ciências da Comunicação. Professor do Departamento de Ciência da Informação da Universidade Estadual Paulista "Júlio de Mesquita Filho" (UNESP). E-mail: guima@marilia.unesp.br
\end{abstract}


internacionais, salientam-se grupos de interesses temáticos, como dimensões éticas e sociais, aspectos bibliométricos, interlocução com as ciências exatas e tecnologias, dentre outros. Conclusões: A liderança do grupo de autores confirma que a revista é aberta a temas como os aspectos éticos e sociais da OC, além das perspectivas tecnológicas no contexto desse domínio. Além disso, uma forte incidência de artigos filiados entre UNESP e UFMG revelou a especificidade e a tradição de pesquisa dessas duas universidades nas temáticas ligadas à organização do conhecimento.

Descritores: Análise de Domínio. Comunidades Epistêmicas. Produção científica brasileira. Knowledge Organization.

\section{INTRODUÇÃO}

A Análise de Domínio (AD), cuja inserção na Ciência da Informação deuse a partir do artigo seminal de Hjørland e Albrechtsen (1995), pode ser considerada atualmente uma importante forma de visualização e compreensão da ciência, sob perspectivas relativas a não apenas um único indivíduo, mas na socialização do conhecimento entre um grupo que compartilha interesses em comum.

Como destaca Smiraglia (2012), um domínio consiste num grupo com uma ontologia compartilhando a mesma epistemologia, isso propicia a identificação das correntes teóricas de uma determinada área e as relações estabelecidas por meio de seus autores.

Uma forma de analisar os domínios de conhecimento é por meio da indicação de comunidades epistêmicas, ou seja, aqueles pesquisadores que investigam sobre determinada temática, publicam sobre ela de maneira assídua, tornando-se referência nesses conteúdos e dessa forma, influenciando novos pesquisadores que desejam se aprofundar nesses assuntos.

Diante disso, a presente pesquisa buscou identificar qual é a comunidade epistêmica brasileira da Organização do Conhecimento, especificamente nas publicações do periódico Knowledge Organization no século XXI, como subsídio à pesquisa na área, em especial no que tange aos principais pesquisadores no âmbito brasileiro que evidenciaram inserção internacional. Além disso, pretende-se popularizar o conceito de comunidades epistêmicas, ainda pouco utilizado na Ciência da Informação. 
Desse modo, parte-se de uma abordagem introdutória sobre análise de domínio para, em seguida, adentrar mais especificamente nas comunidades epistêmicas, de modo a embasar teoricamente a pesquisa, seguindo-se à descrição da metodologia, e à apresentação de discussão dos resultados de modo a subsidiar as conclusões.

\section{ANÁLISE DE DOMÍNIO}

Hjorland e Albrechtsen (1995) em seu seminal artigo Toward a New Horizon in information Science: Domain-Analysis, apresentam a Análise de Domínio como um caminho para a compreensão dos diversos domínios da Ciência da Informação, subsidiando a caracterização de seus processos de construção e de socialização do conhecimento.

O conceito de AD foi posteriormente estruturado por Hjorland (2002), que propôs onze abordagens, a saber: Produção de obras de referência, Construção de linguagens de indexação, Indexação e recuperação da informação, Estudo de usuários, Estudos bibliométricos, Estudos históricos, Estudos de gêneros/tipologias documentais, Estudos epistemológicos e críticos, Estudos terminológicos, Comunicação científica, Cognição científica, conhecimento especializado e inteligência artificial. Pode-se incluir também a esse grupo o da Proveniência Arquivística proposto pelos pesquisadores Guimarães e Tognoli (2015).

Hoje se verifica, nesse âmbito, uma forte predominância de duas abordagens, muitas das vezes tratadas conjuntamente: a abordagem bibliométrica (MOYA-ANEGÓN; HERRERO-SOLANA, 2001; SMIRAGLIA, 2012) e a abordagem epistemológica (HJØRLAND, 2002; TENNIS, 2003), o que possibilita identificar as temáticas de um determinado grupo como também as relações estabelecidas por tais, em diversos âmbitos: científico, psicossocial e sociolinguístico (HJORLAND; ALBRECHTSEN, 1995).

Buscando um enfoque mais operacional, Tennis (2003) propõe dois eixos para a efetivação da análise de domínio: as áreas de modulação (denominação e limite do domínio) e os graus de especialização (especificidade do domínio). 
Especificamente no que tange à abordagem bibliométrica, objeto desta pesquisa, destaca Hjorland (2002) o seu papel instrumental para o mapeamento do estado da arte de um determinado campo de pesquisa, seja por domínio, instituição ou país, além disso, possibilita identificar colaborações de autores e instituições (coautorias) que permitir detectar a produção científica e os grupos com interesses temáticos em comum, como também o nível de associação que pode ser regional, nacional e internacional (GRAClO; OLIVEIRA, 2011).

Nesse contexto, a abordagem bibliométrica da análise de domínio, ao fornecer elementos para o estudo da produção e uso do conhecimento, elucida questões sobre o universo (domínio), as temáticas tratadas e o universo de atores (autores ou referentes) que aí interagem e que se agrupam naquilo que se pode denominar como comunidades epistêmicas.

\section{COMUNIDADES EPISTÊMICAS}

Embora o conceito de comunidades epistêmicas tenha sido preconizado por Burkart Holzner, em sua obra Reality Construction in Society de 1968, foi notadamente a partir de 1992, com um fascículo especial da revista International Organization que ele foi de fato popularizado. Nesse número, Peter Haas, importante teórico do tema, busca conceituar o termo e definir limites do que ele abrange. Segundo o autor, comunidades epistêmicas podem ser definidas enquanto

[...] uma rede de profissionais com reconhecida expertise e competência em um domínio em particular, com autoridade e conhecimento relevante em políticas dentro desse domínio ou área (HAAS, 1992, p. 03, tradução nossa).

Para Haas, essa abordagem de análise permite identificar como redes de especialistas em determinado domínio agem diante dos problemas enfrentados pela área, confirmando o poder implícito no controle do conhecimento e da informação, além de demonstrar novos padrões de comportamento verificados na difusão de novas ideias.

De acordo com o autor, para compor uma comunidade epistêmica, os 
autores devem partilhar uma série de preceitos, dentre eles: um conjunto de normativas e princípios; crenças causais derivadas da prática; noções de validade, de forma a validar o conhecimento no domínio e uma política comum, visando o bem-estar humano (HAAS, 1992).

Ainda nesse número da International Organization, Adler (1992) também busca conceituar o termo, afirmando que comunidades epistêmicas podem ser utilizadas para organizar, estruturar e coordenar a colaboração internacional, por meio de práticas comuns e que visam promover a mudança. Essas comunidades são desenvolvidas em encontros internacionais, em que pesquisadores de prestígio se reúnem para discutir perspectivas para problemas em comum.

Adler (1992) ainda afirma que para que essas comunidades sejam bem sucedidas, é necessário que alguns aspectos sejam levados em conta, como por exemplo o contingente histórico influenciado pelas tecnologias proporcionadas, distribuição de poder, políticas nacionais, estruturas administrativas, economia, dentre outros.

Para o autor, essas comunidades serão compostas basicamente por associações informais entre cientistas e profissionais, que por razões intelectuais, ideológicas ou políticas adotaram abordagens similares para resolução de um problema, apesar das possíveis diferenças entre os membros. Os participantes da comunidade conhecem-se bem, encontram-se frequentemente em debates e mesas-redondas e, em alguns casos, são colegas em universidades próximas e aprenderam um com o outro sobre as questões pesquisadas.

Levando em consideração essas questões, o presente trabalho buscou analisar como domínio a produção brasileira sobre Organização do Conhecimento no âmbito específico do periódico Knowledge Organization, de modo a estabelecer uma comunidade epistêmica nesse âmbito. Os passos metodológicos desenvolvidos pela pesquisa encontram-se descritos na próxima seção. 


\section{METODOLOGIA}

Conforme mencionado anteriormente, a presente pesquisa adota como metodologia a Análise de Domínio, difundida na Ciência da Informação por Hjørland e Albrechtsen (1995). Para tanto, utilizou-se de duas das abordagens dessa metodologia (Hjørland, 2002): prioritariamente, os estudos bibliométricos e, de forma subsidiária, as estruturas de comunicação científica (décima abordagem).

De acordo com o referido autor, os estudos bibliométricos são uma importante forma de análise de domínio, pois possibilitam a visualização das conexões na ciência por meio dos documentos. Por meio desses estudos, verifica-se o que o autor denomina como "dependência" entre pesquisadores, instituições, campos, abordagens e localizações geográficas.

Os estudos sobre as estruturas de comunicação científica, por sua vez, auxiliam na construção de manuais sobre determinadas disciplinas, além de informar e guiar pessoas de fora do domínio. Essas características ficaram claras durante o desenvolvimento da presente pesquisa ao estabelecer a comunidade epistêmica do domínio estudado.

A fonte de pesquisa foi o periódico Knowledge Organization, criado por Ingetraut Dahlberg, que também foi idealizadora da International Society for Knowledge Organization (ISKO), em 1973, tendo seu primeiro número publicado em 1974. Vale destacar que a referida revista, publicada ininterruptamente desde sua criação, constitui o mais abalizado periódico científico na área de organização do conhecimento em âmbito mundial.

Inicialmente, denominado International Classification, o referido periódico tornou-se veículo científico oficial da ISKO em 1989 e, em 1993, passou a ter a denominação atual. De acordo com o site oficial, a revista preocupa-se com temáticas voltadas para questões teóricas, aspectos práticos dos processos e ferramentas, configurações históricas, educação e treinamento e questões terminológicas, todos voltados para a área de Organização do Conhecimento (ISKO, 2019).

Para compor o corpus, foram selecionados os trabalhos que 
contivessem ao menos um autor brasileiro ou afiliado a alguma instituição brasileira. Como período temporal, optou-se pelos anos entre 2001-2018, ou seja, aqueles publicados no século XXI. Para tanto, verificou-se, a partir dos sumários dos números publicados nesse período, quais encaixavam-se no contexto brasileiro descrito.

Dessa forma, foi recuperado um total de 51 artigos, que foram sistematicamente analisados de maneira a identificar autores mais produtivos, instituições com maior incidência, coautorias internacionais e anos de maior publicação. Os resultados podem ser verificados na sessão seguinte.

\section{RESULTADOS}

O primeiro aspecto verificado foi o estudo cronológico, ou seja, quantidade de artigos por ano. Essa informação pode ser melhor analisada no gráfico a seguir:

Figura 1 - Quantidade de artigos por ano

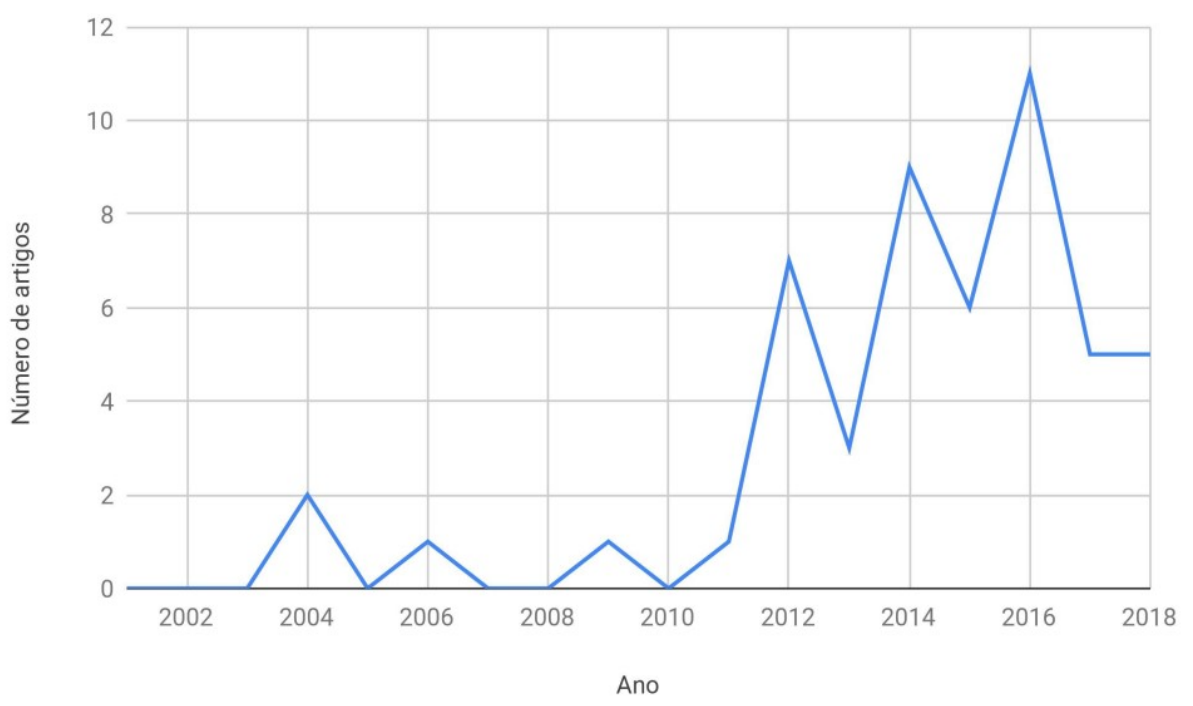

Fonte: Elaborado pelos autores (2019)

No gráfico estão descritos apenas os anos em que houve alguma produção brasileira na revista. Sendo assim, é possível perceber que nos anos de 2001, 2002, 2003, 2005, 2007, 2008 e 2010 não houve nenhum artigo que se encaixasse nos parâmetros definidos pela pesquisa e nos anos que 
intercalam esse período descrito, a produção foi relativamente baixa: 1 ou 2 artigos por ano.

Dessa forma, identifica-se um considerável crescimento nos anos mais recentes - a partir de 2011 , sendo que $85 \%$ do total de publicações brasileiras na revista foi nesse período. Esse fator demonstra que o periódico vem se popularizando no contexto brasileiro nos últimos anos.

É interessante observar que essa maior produção brasileira se verifica a partir da realização do I Congresso Brasileiro de Organização do Conhecimento, realizado em 2011, reflexo da criação do capítulo brasileiro da ISKO em 2007, o que permitiu um espaço agregador dos pesquisadores nessa temática e uma maior interlocução desses com a ISKO internacional.

Esse total de publicações foi dividido entre 72 autores, no entanto, um grupo deles se destaca, como pode ser verificado na tabela a seguir:

Tabela 1 - Autores mais produtivos

\begin{tabular}{ll}
\hline Autor & $\begin{array}{l}\text { Número de } \\
\text { autorias }\end{array}$ \\
\hline GUIMARÃES, J. A. C. & 07 \\
\hline MARTINEZ-ÁVILA, D. & 06 \\
\hline PINHO, F. A. & 05 \\
\hline SOUZA, R. R. & 04 \\
\hline LIMA, G. Â. B. & 03 \\
\hline ALMEIDA, C. C. & 03 \\
\hline FUJITA, M. S. L. & 03 \\
\hline MOURA, M. A. & 03 \\
\hline ALVARENGA, L. & 02 \\
\hline ALMEIDA, M. B. & 02 \\
\hline ORTEGA, C. D. & 02 \\
\hline MILANI, S. O. & 02 \\
\hline CAMPOS, M. L. A. & 02 \\
\hline GOMES, H. E. & 02 \\
\hline MARCONDES, C. H. & 02 \\
\hline ARBOIT, A. E. & 02 \\
\hline TOGNOLI, N. B. & 02 \\
\hline SALDANHA, G. S. & 02 \\
\hline CASTANHA, R. C. G. & 02 \\
\hline
\end{tabular}

Fonte: Elaborado pelos autores (2019)

Nessa tabela, constam os autores que tiveram ao menos 02 trabalhos publicados na revista no período analisado. Sendo assim, um grupo de 53 autores $(74 \%)$ tiveram apenas uma incidência de publicação na revista. A comunidade epistêmica brasileira na revista caracteriza-se, portanto, por $26 \%$ dos autores que já tiveram trabalhos divulgados pela revista, um total de 19 
pesquisadores.

Desse grupo, é relevante afirmar que Gercina Ângela Borém Lima, da Universidade Federal de Minas Gerais (UFMG), foi a primeira pesquisadora brasileira a ter um artigo publicado pela revista, em 2004. Outro destaque que deve-se a Daniel Martínez-Ávila, figurado em segundo lugar na lista, pesquisador espanhol cuja produção foi contabilizada apenas a partir de 2014, ano em que se radicou na Universidade Estadual Paulista (UNESP).

Observa-se ainda que quatro autores, nomeadamente José Augusto Chaves Guimarães, Daniel Martínez-Ávila, Fábio de Assis Pinho e Renato Rocha de Souza respondem por $31 \%$ das autorias brasileiras na revista e $40 \%$ das autorias desse grupo selecionado.

Destaca-se ainda a forte interlocução entre os três primeiros pesquisadores na tabela, que possuem pesquisas em comum e publicam majoritariamente sobre questões de representatividade dos grupos marginalizados nas ferramentas de OC e aspectos teóricos em ética em OC, além de análises sobre a cobertura desse tema na literatura da área.

Além de autores mais produtivos, verificou-se também as universidades que mais representaram o contexto brasileiro na revista. Deve-se ter em mente que, em alguns casos, há a ocorrência de colaborações interinstitucionais em um mesmo artigo e portanto, a incidência de universidades é maior que o número de artigos. Os dados encontram-se descritos na próxima tabela:

Tabela 2 - Instituições mais produtivas

\begin{tabular}{ll}
\hline Instituições & $\begin{array}{l}\text { Número de } \\
\text { autorias }\end{array}$ \\
\hline Universidade Estadual Paulista (UNESP) & 22 \\
\hline $\begin{array}{l}\text { Universidade Federal de Minas Gerais } \\
\text { (UFMG) }\end{array}$ & 14 \\
\hline $\begin{array}{l}\text { Universidade Federal de Pernambuco } \\
\text { (UFPE) }\end{array}$ & 05 \\
\hline Universidade Federal Fluminense (UFF) & 04 \\
\hline $\begin{array}{l}\text { Instituto Brasileiro de Informação em } \\
\text { Ciência e Tecnologia (IBICT) }\end{array}$ & 04 \\
\hline Fundação Getúlio Vargas (FGV) & 02 \\
\hline $\begin{array}{l}\text { Universidade Federal do Rio de Janeiro } \\
\text { (UFRJ) }\end{array}$ & 02 \\
\hline Fiocruz & 02 \\
\hline $\begin{array}{l}\text { Universidade Federal de Santa Catarina } \\
\text { (UFSC) }\end{array}$ & 02 \\
\hline
\end{tabular}

Fonte: Elaborada pelos autores (2019) 
Da mesma forma que na tabela anterior, nesta estão descritas aquelas instituições que estiveram presentes nas publicações ao menos duas vezes. Salienta-se que apenas no caso da Universidade Federal de Pernambuco (UFPE), a contribuição veio de um único autor - professor Fábio de Assis Pinho. Nos outros casos, as contribuições vieram de autores diversos, inclusive daqueles que publicaram uma única vez na revista.

Percebe-se que há uma forte liderança compartilhada pelas universidades Universidade Estadual Paulista (UNESP) e Universidade Federal de Minas Gerais (UFMG) que, do total de 67 incidências institucionais, apareceram 36 vezes, ou seja, $54 \%$ do total. Isso revela a especificidade e a tradição de pesquisa dessas duas universidades nas temáticas ligadas à organização do conhecimento.

É importante ressaltar que essas duas universidades de destaque são também, as universidades que os quatro primeiros autores mais produtivos fazem ou fizeram parte, destacando mais uma vez que o periódico possui especial interesse com os aspectos sociais e tecnológicos da representação da informação e que o Brasil pode ser considerado um importante produtor de ciência nesse sentido.

Destaca-se também a presença não apenas de universidades, mas de institutos de pesquisa, como Instituto Brasileiro de Informação em Ciência e Tecnologia - IBICT (com 04 artigos) e Fiocruz (com 02 artigos). Além disso, há a predominância de instituições públicas, com uma instituição particular figurando na lista (Fundação Getúlio Vargas - FGV).

É interessante notar ainda, a presença de 8 universidades $^{1}$ estrangeiras responsáveis por coautorias internacionais, em especial University of Wisconsin-Milwaukee (EUA), com 04 publicações em coautoria com pesquisadores brasileiros e University of Madras (Índia), com 02 trabalhos de autoria de K. S. Raghavan em parceria com autores brasileiros.

\footnotetext{
${ }^{1}$ As universidades em que houveram coautorias internacionais são, além das duas citadas: Pennsylvania State University, University of Glamorgan, Instituto Politécnico do Porto, Universidad de Zaragoza, University of Western Ontario e Brunel University London, cada uma com uma incidência.
} 
Além disso, destaca-se os países dos quais tiveram colaborações com os autores brasileiros, sendo que, a maior contribuição vem da parceira com os EUA com 5 artigos publicados, depois Reino Unido e Índia com 2 artigos cada, seguido por 1 artigo que tem a colaboração da Espanha e Portugal, e por último 1 artigo com o Canadá, totalizando assim 11 publicações com coautorias internacionais o que, corresponde a $22 \%$ de toda a produção científica no período analisado.

Relativamente às coautorias nacionais, algumas relações devem ser destacadas. Para tanto, foi elaborada uma rede, que pode ser melhor visualizada no Apêndice A. A partir dessa rede, alguns grupos de interesse de pesquisa e suas relações intertemáticas merecem ser evidenciadas.

Foi possível identificar um forte grupo que trabalha com as relações sociais e estudos críticos em organização do conhecimento, possuindo José Augusto Guimarães e Daniel Martínez-Ávila (UNESP) como epicentros, grupo esse que também se encontra ligado a um grupo preocupado com questões mais voltadas aos aspectos epistemológicos do conhecimento. Outro grupo interdisciplinar que merece destaque é aquele liderado por Mariângela Fujita (UNESP), com artigos voltados para análise de sistemas de organização do conhecimento, mas que também se encontra relacionado aos aporte semióticos nesse contexto.

Outros grupos de interesse puderam ser identificados, nomeadamente: a) relativo aos estudos em recuperação da informação, ontologias e hipertexto, liderados por Gêrcina Ângela Borém Lima (UFMG); b) relativo aos estudos em web semântica e taxonomias, que possuem Renato Rocha Souza (UFMG) como pesquisador central; c) relativo às pesquisas em metadados, ontologias e tesauro, liderados por Lídia Alvarenga (UFMG); e d) relativos aos aspectos semânticos da organização do conhecimento, em que Maria Aparecida Moura (UFMG) figura como pesquisadora central. Além disso, foi possível perceber uma relação bem distribuída, composta por Maria Luiza de Almeida Campos, Laura de Lira e Oliveira, Hagar Espanha Gomes (UFF), Maria Luiza Machado Campos (UFRJ) e Albeto Martín Rivera Dávila (Fiocruz), que dedicam-se a estudar a representação do conhecimento e ontologias. 
Algumas relações bilaterais também puderam ser identificadas, como Silva e Ortega, que se preocuparam com os estudos históricos em arranjos de organização do conhecimento; Gustavo Saldanha e Naira Silveira (IBICT/UNIRIO), responsáveis por estudos filosóficos na área; Renata Castanha e Maria Cláudia Grácio (UNESP), com interesse nos aspectos bibliométricos e em análise de domínio; e Leonardo Costa e Carlos Marcondes (UFF), com estudos em web semântica.

De maneira semelhante, fez-se necessário demonstrar as coautorias internacionais identificadas nos artigos analisados. Também para uma melhor visualização, a rede bibliométrica resultante dessas relações foi incluída no Apêndice B.

Nessa rede, é possível verificar grupos de interesse de pesquisa fortemente ligados, com assuntos que giram em torno das dimensões sociais da organização do conhecimento, aspectos bibliométricos nesse domínio, como também de representação e recuperação do conhecimento no âmbito da tecnologia no que tange os temas de arquitetura da informação, banco de dados, web semântica.

É perceptível também que, os autores José Augusto Chaves Guimarães (UNESP), Daniel Martínez-Ávila (UNESP) e Renato Rocha Souza (UFMG) estão nos epicentros de conexões entre autores brasileiros e autores internacionais.

Esse cenário demonstra a centralidade institucional da UNESP e da UFMG nas pesquisas nesse domínio de conhecimento, seja na produção em coautorias nacionais seja na colaboração internacional.

Destaca-se também, em específico, um grupo de interesse que discute as questões de organização do conhecimento sob viés da tecnologia, em que os autores Márcio Victorino, Maristela Terto de Holanda, Edison Ishikawa, Edgard Costa Oliveira (UnB) são advindos das áreas de Ciência da Computação e de Engenharia, e contribuem para a Ciência da Informação de forma relevante, em um efetivo diálogo interdisciplinar. 


\section{CONCLUSÕES}

O presente trabalho buscou demonstrar de que forma se apresenta 0 contexto brasileiro no universo do periódico Knowledge Organization, importante revista no âmbito da Organização do Conhecimento. A partir desse contexto, pretendeu-se estabelecer qual é a comunidade epistêmica brasileira nesse domínio, que muitas vezes é utilizado como instrumento de pesquisa para aqueles investigadores que buscam iniciar ou complementar seus estudos na área.

Embora a escolha de uma única fonte de pesquisa possa ser considerada uma fragilidade na pesquisa, ressalta-se que, conforme evidenciado anteriormente e comprovado durante a pesquisa, o periódico analisado representa de maneira profícua o domínio da Organização do Conhecimento, produzindo um corpus de pesquisa de número considerável e em que foi possível realizar uma análise sistemática.

A partir dos resultados, foi possível identificar uma comunidade epistêmica constituída de 19 autores (apresentados na Tabela 01) e, mais especificamente, liderada por 4 pesquisadores que produzem constantemente sobre seus temas de pesquisa, de maneira a servir como fonte e também influenciar consistentemente as pesquisas realizadas em suas temáticas.

A produção de duas universidades - UNESP e UFMG - também merece ser evidenciada, uma vez que essas duas instituições aparecem em quase metade das afiliações institucionais de toda a produção brasileira no periódico, podendo inferir-se que há um incentivo e investimento em publicações internacionais de renome, como a revista analisada.

Esse incentivo pode ser verificado a partir da popularização das publicações brasileiras no periódico nos últimos anos, em que houve um crescimento exponencial. Essa ascensão auxilia na disseminação da ciência brasileira em âmbito internacional, uma vez que os artigos são escritos no idioma inglês, o que amplia a capacidade de compreensão de pesquisadores estrangeiros. Reitera-se, aqui, a importância da criação e da atuação do capítulo brasileiro da ISKO para a internacionalização da pesquisa brasileira 
em organização do conhecimento, com especial destaque para os quatro congressos brasileiros de organização do conhecimento até então realizados (Brasília, 2011; Rio de Janeiro, 2013; Marília, 2015 e Recife, 2017), ocasião em que se pôde contar com a presença de renomados pesquisadores internacionais assim como se pôde divulgar mais especificamente a produção brasileira, como é o caso dos anais do evento de 2015, que teve uma edição totalmente em inglês (2017) seja por conta de muitos pesquisadores

Outro fator que deve ser considerado são os grupos temáticos verificados na rede, com contribuições internacionais, o que também demonstra uma interlocução entre a produção brasileira de ciência e pesquisas desenvolvidas em outros países. Essa interlocução, além de também auxiliar na disseminação das universidades brasileiras enquanto centros de pesquisa, igualmente cria um ambiente de discussão multicultural e rico em experiências diversas.

A vista dessas questões, recomenda-se a realização de investigações futuras neste sentido, relativamente à internacionalização da pesquisa brasileira em organização do conhecimento, em espaços mais específicos como os anais dos congressos internacionais da ISKO, nos congressos dos capítulos nacionais e/ou regionais da ISKO ou, ainda, em veículos externos à ISKO, tais como as revistas Journal of Documentation, The indexer, JASIST, Journal of Information e Scire, que igualmente vêm, dedicando espaço privilegiado à pesquisa em organização do conhecimento.

Agradecimentos: A autora Isadora Victorino Evangelista agradece 0 financiamento por meio do processo n 2017/02327-8, Fundação de Amparo à Pesquisa do Estado de São Paulo (FAPESP). A autora Manoela Ferreira da Silva agradece 0 financiamento por meio do processo $n^{\circ}$ 145989/2018-6, Programa Institucional de Bolsas de Iniciação Científica (PIBIC/CNPq). O autor José Augusto Chaves Guimarães agradece o financiamento por meio do processo nº 2017/00584-3, Fundação de Amparo à Pesquisa do Estado de São Paulo (FAPESP). 


\section{REFERÊNCIAS}

ADLER, Emanuel. The emergence of cooperation: national epistemic communities and the international evolution of the idea of nuclear arms control. International Organization, v. 46, n. 1, 1992, p. 101-145.

GRACIO, Maria Cláudia Cabrini; OLIVEIRA, Ely Francina Tannuri de. Produção e comunicação da informação em CT\&I - GT7 da ANCIB: análise bibliométrica no período 2003/2009. Liinc em Revista, Rio de Janeiro, v. 7, n. 1, mar. 2011, p. 248-263.

GUIMARÃES, José Augusto Chaves; TOGNOLI, Natália Bolfarini. Provenance as a domain analysis approach in archival knowledge organization. Knowledge Organization, v. 42, n. 8, 2015, p. 562-569.

HAAS, Peter M. Epistemic communities and international policy coordination. International Organization, Cambridge, v. 46, n. 1, p. 01-35, 1992.

HJøRLAND, Birger; ALBRECHTSEN, Hanne. Toward a new horizon in information science: domain-analysis. Journal of the American Society for Information Science, v. 46, n. 6, 1995, p. 400-425.

HJøRLAND, Birger. Domain analysis in information Science: eleven approaches traditional as well as innovative. Journal of Documentation, v. 58, n. 4,2002 , p. $422-462$.

MOYA-ANEGÓN, Felix de; HERRERO-SOLANO, Víctor. Análisis de dominio de la revista mexicana de investigación bibliotecológica. Información, cultura y sociedad, v. 5,2001, p. $10-28$

SMIRAGLIA, Richard P. Epistemology of Domain Analysis. In: LEE, H-L.; SMIRAGLIA, R.P. (ed.). Cultural frames of knowledge. Wurzburg: Ergon, 2012. p.111-124.

TENNIS, Joseph T. Two Axes of Domain Analysis. Knowledge Organization, v. 30, n. 3/4, 2003, p. 191-195.

\section{BIBLIOGRAFIA ANALISADA}

ALMEIDA, Carlos Cândido de. The Methodological Influence of Peirce's Pragmatism 
on Knowledge Organization. Knowledge Organization, v. 39, n. 03, p. 204-215, 2012.

ALMEIDA, Carlos Cândido; FUJITA, Mariângela Spotti Lopes; REIS, Daniela Marjorie . Peircean semiotics and subject indexing: contributions of speculative grammar and pure logic. Knowledge Organization, v. 40, n. 04, p. 225-241, 2013.

ALMEIDA, Maurício; SOUZA, Renato; FONSECA, Fred. Semantics in the semantic web: a critical evaluation. Knowledge Organization, v. 38, n. 03, p. 187-203, 2011.

ANDRADE, Julietti; LARA, Marilda Lopes Ginez de. Interoperability and mapping between Knowledge Organization Systems: Metathesaurus-Unified Medical Language System of the National Library of Medicine. Knowledge Organization, v. 43, n. 02, p. 107-112, 2016.

ARBOIT, Aline Elis; GUIMARÃES, José Augusto Chaves. The ethics of knowledge organization and representation from a Bakhtinian perspective. Knowledge Organization, v. 42, n. 05, p. 324-331, 2015.

ARBOIT, Aline Ellis. Knowledge Organization: From Term to Concept, From Concept to Domain. Knowledge Organization, v. 45, n. 2, p. 125-136, 2018.

BRANDT, Mariana Baptista. Ethical Aspects in the Organization of Legislative Information. Knowledge Organization, v. 45, n. 1, p. 3-12, 2018.

BRASCHER, Marisa. Semantic Relations in Knowledge Organization Systems. Knowledge Organization, v. 41, n. 02, p. 175-180, 2014.

CAFÉ, Lígia Maria Arruda; SOUZA, Renato Rocha. Sentiment Analysis and Knowledge Organization: An Overview of the International Literature. Knowledge Organization, v. 44, n. 3, p. 199-214. 2017.

CAMPBELL, D. Grant; GUIMARÃES, José Augusto Chaves; PINHO, Fabio Assis; MARTÍNEZ-ÁVILA, Daniel; NASCIMENTO, Francisco Arrais. The Terminological Polyhedron in LGBTQ Terminology: Self-Naming as a Power to Empower in Knowledge Organization. Knowledge Organization, v. 44, n. 8, p. 586-591, 2017.

CAMPOS, Maria Luiza de Almeida; CAMPOS, Maria Luiza Machado; DÁVILA, Alberto m. R.; GOMES, Hagar Espanha; OLIVEIRA, Laura de Lira. Information sciences methodological aspects applied to ontology reuse tools: a study based on genomic annotations in the domain of trypanosomatides. Knowledge Organization, v. 40, n. 03, p. 50-61, 2013.

CAMPOS, Maria Luiza de Almeida; GOMES, Hagar Espanha. Ontology: Several 
Theories on the Representation of Knowledge Domains. Knowledge Organization, v. 44, n. 3, p. 178-186, 2017.

CASTANHA, Renata Cristina Gutierres; WOLFRAM, Dietmar. The Domain of Knowledge Organization: A Bibliometric Analysis of Prolific Authors and Their Intellectual Space. Knowledge Organization, v. 45, n. 1, p. 13-22, 2018.

CASTANHA, Renata Cristina Gutierrez; Gracio, Maria Cláudia Cabrini. Bibliometrics contribution to the metatheoretical and Domain Analysis Studies. Knowledge Organization, v. 41, n. 02, p. 171-174, 2014.

COLLOVINI DE ABREU, Sandra; VIEIRA, Renata. ReIP: Portuguese Open Relation Extraction. Knowledge Organization, v. 44, n. 3, p. 163-177, 2017.

DE SANTIS, Rodrigo; SOUZA, Rosali Fernandez. Classifying popular songs: possibilities and challenges. Knowledge Organization, v. 41, n. 02, p. 181-187, 2014.

FREITAS, Juliana Lazarotto; GABRIEL JUNIOR, Rene Faustino; BUFRÉM, Leilah Santiago. Theoretical approximations between Brazilian and spanish authors' production in the field of Knowledge Organization in the production of journals on Information Science in Brazil. Knowledge Organization, v. 39, n. 03, p. 216-223, 2012.

FUJITA, Mariângela Spotti Lopes, MOREIRA, Walter; SANTOS, Luciana Beatriz Piovezan dos; ANDRADE E CRUZ, Maria Carolina; de BARROS RIBAS, Rosane Rodrigues. Construction and Evaluation of Hierarchical Structures of Indexing Languages for Online Catalogs of Libraries: An Experience of the São Paulo State University (UNESP). Knowledge Organization, v. 45, n. 3, p. 220-231, 2018.

GUEDES, Roger de Miranda; MOURA, Maria Aparecida. The principle of semantic warrant and the study of language: conceptual reflections. Knowledge Organization, v. 43, n. 02, p. 102-106, 2016.

GUIMARÃES, José Augusto Chaves; PINHO, Fabio Assis; MILANI, Suellen Oliveira. Theoretical Dialogs About Ethical Issues in Knowledge Organization: García Gutiérrez, Hudon, Beghtol, and Olson. Knowledge Organization, v. 43, n. 5, p. 338-350, 2016.

GUIMARÃES, José Augusto Chaves; TOGNOLI, Natália Bolfarini. Provenance as a Domain Analysis approach in Archival Knowledge Organization. Knowledge Organization, v. 42, n. 08, p. 562-569, 2015.

LIMA, Gercina Ângela Borém; RAGHAVAN, K. S. Information Retrieval and Cognitive Research. Knowledge Organization, v.31, n. 2, p. 98-105, 2004. 
LOURENÇO, Cíntia de Azevedo; ALVARENGA, Lídia. Metadata Standard of Theses and Dissertations according to the Entity-Relationship Model. Knowledge Organization, v.36, n. 1, p. 30-45, 2009.

MARCONDES, Carlos H.; COSTA, Leonardo C. A model to represent and process scientific knowledge in biomedical articles with semantic web technologies. Knowledge Organization, v. 43, n. 02, p. 269-275, 2016.

MARCONDES, Carlos Henrique. Knowledge Organization and representation in digital environments: relations between ontology and knowledge organization. Knowledge Organization, v. 40, n. 02, p. 115-122, 2013.

MARTELETO, Regina Maria; CARVALHO, Lidiane dos Santos. The representation of ethics and knowledge organization in the WoS and LISTA databases. Knowledge Organization, v. 42, n. 08, p. 581-590, 2015.

MARTÍNEZ-ÁVILA, Daniel. Knowledge Organization in the intersection with information technologies. Knowledge Organization, v. 42, n. 07, p. 486-498, 2015.

MARTÍNEZ-ÁVILA, Daniel; BEAK, Jihee. Methods, Theoretical Frameworks and Hope for Knowledge Organization. Knowledge Organization, v. 43, n. 5, p. 358-366., 2016.

MARTÍNEZ-ÁVILA, Daniel; GUIMARÃES, José Augusto Chaves; PINHO, Fábio de Assis; FOX, Melodie J. The representation of ethics and knowledge organization in the WoS and LISTA databases. Knowledge Organization, v. 42, n. 05, p. 269-275, 2015.

MARTÍNEZ-ÁVILA, Daniel; KIPP, Margaret E.I. Implications of the Adoption of BISAC for Classifying Library Collections. Knowledge Organization, v. 41, n. 5, n. 377-392, 2014.

MARTÍNEZ-ÁVILA, Daniel; SEMIDÃO, Rafael; FERREIRA, Marcio. Methodological Aspects of Critical Theories in Knowledge Organization. Knowledge Organization, v. 43, n. 2, p. 118-125, 2016.

MEIRELES, Magali Rezende Gouvêa; CENDÓN, Beatriz Valadares; ALMEIDA, Paulo Eduardo Maciel de. Bibliometric Knowledge Organization: A Domain Analytic Method Using Artificial Neural Networks. Knowledge Organization, v. 41, n. 2, p.45-159.

MILANI, Suelen de Oliveira; PINHO, Fábio de Assis. Knowledge representation and orthophemism: a reflection aiming to a concept. Knowledge Organization, v. 39, n. 05, p. 384-393, 2012.

MONTEIRO, Silvana Drumond; MOURA, Maria Aparecida. Knowledge Graph and 
"Semantization" in Cyberspace: a study of contemporary indexes. Knowledge Organization, v. 41, n. 06, p. 429-439, 2014.

MOREIRA, Alexandra; ALVARENGA, Lídia; OLIVEIRA, Alcione de Paiva. "Thesaurus" and "Ontology:" A Study of the Definitions Found in the Computer and Information Science Literature, by Means of an Analytical Synthetic Method. Knowledge Organization, v. 31, n. 4, p. 231-244, 2004.

MOURA, Maria Aparecida. Emerging Discursive Formations, Folksonomy and Social Semantic Information Spaces (SSIS): the contributions of the Theory of Integrative Levels in the studies carried out by the Classification Research Group (CRG). Knowledge Organization, v. 41, n. 04, p. 304-310, 2014.

NETTO, Cristiane; de OLIVEIRA LIMA, Gêrcina Ângela Borém; PIEROZZI JÚNIOR, Ivo. An Application of Facet Analysis Theory and Concept Maps for faceted search in domain ontology: preliminary studies. Knowledge Organization, v. 43, n. 4, p. 254-264, 2016.

ORTEGA, Cristina Dotta. Conceptual and procedural grounding of documentary systems. Knowledge Organization, v. 39, n. 03, p. 224-228, 2012.

PANDO, Daniel Abraão; ALMEIDA, Carlos Cândido de. Knowledge Organization in the context of postmodernity from the Theory of Classification perspective. Knowledge Organization, v. 43, n. 02, p. 113-117, 2016.

PINHO, Fábio de Assis; GUIMARÃES, José Augusto Chaves. Male homosexuality in brazilian indexing languages: some ethical questions. Knowledge Organization, v. 39, n. 05, p. 363-369, 2012.

PINTO, Virgínia Bentes; RABELO, Camila Regina de Oliveira; GIRÃO, Igor Peixoto Torres. SNOMED-CT as Standard Language for organization and representation of the information in patient records. Knowledge Organization, v. 41, n. 04, p. 304-310, 2014.

RODRIGUES, Fernando de Assis; SANT'ANA, Ricardo César Gonçalves. Use of Taxonomy of Privacy to Identify Activities Found in Social Networks' Terms of Use. Knowledge Organization, v. 43, n. 4, p. 285-295, 2016.

SALDANHA, Gustavo. The Philosophy of Language and Knowledge Organization in the 1930s: Pragmatics of Wittgenstein and Ranganathan. Knowledge Organization, v. 41, n. 04, p. 296-303, 2014.

SILVA, Andrieli Pachú da; GUIMARÃES, José Augusto Chaves; TOGNOLI, Natália Bolfarini. Ethical values in archival arrangement and description: an analysis of professional codes of ethics. Knowledge Organization, v. 42, n. 05, p. 346-352, 2015. 
SILVA, Camila Mariana Aparecida da; ORTEGA, Cristina Dotta. Proposals That Preceded the Call Number: Shelf Arrangement in the Francofone Manuals of Librarianship from the Mid-Nineteenth Century to 1930. Knowledge Organization, v. 44, n. 8, p. 605-614, 2017.

SILVA, Guilherme Baião Salgado; LIMA, Gercina Ângela Borém de Oliveira. Using topic maps in establishing compatibility of semantically structured hypertext contents. Knowledge Organization, v. 39, n. 06, p. 432-445, 2012.

SILVEIRA, Naira Christofoletti; SALDANHA, Gustavo Silva.'Own name' in Knowledge Organization Epistemology: A Philosophical Theoretical Debate. Knowledge Organization, v. 43, n. 4, p. 265-278, 2016.

SOUZA, Renato Rocha; RAGHAVAN, K.S. A methodology for noun phrase-based automatic indexing. Knowledge Organization, v. 33, n. 1, p. 45-56, 2006.

SOUZA, Renato Rocha; TUDHOPE, Douglas; ALMEIDA, Maurício Barcellos. Towards a Taxonomy of KOS: Dimensions for Classifying Knowledge Organization Systems. Knowledge Organization, v. 39, n. 02, p. 187-203, 2012.

TERRA, Ana Lúcia; FUJITA, Mariângela Spotti Lopes; AGUSTíN-LACRUZ; María del Carmen. School Libraries and Indexing Policies in Brazil and Portugal. Knowledge Organization, v. 43, n. 4, p. 279-284, 2016.

VICTORINO, Marcio; HOLANDA, Maristela Terto de; ISHIKAWA, Edison; OLIVEIRA, Edgard Costa; CHHETRI, Sammohan. Transforming Open Data to Linked Open Data Using Ontologies for Information Organization in Big Data Environments of the Brazilian Government: the Brazilian Database Government Open Linked Data DBgoldbr. Knowledge Organization, v. 45, n. 6, p. 443-466, 2018.

\title{
THE PRESENCE OF BRAZILIAN SCIENTIFIC PRODUCTION IN THE KNOWLEDGE ORGANIZATION JOURNAL ON 21ST CENTURY
}

\begin{abstract}
Introduction: The Knowledge Organization journal is today one of the main vehicles of scientific communication with regard to Knowledge Organization (OC) issues. Objetive: Thus, it was considered relevant to analyze how the Brazilian context is presented in these communications, verifying which authors and institutions are most productive, as well as frequency of publications, national coauthors and international collaborations. Methodology: Through the analysis of the numbers published in the period 2001-2018, it was possible to identify a strong presence of articles of Brazilian authorship, with a total of 51 productions. Results: Was found a community led by Professors José Augusto Chaves Guimarães, Daniel Martínez-Ávila, Fábio de Assis Pinho and Renato Rocha de Souza, who are responsible for $28 \%$ of all publications in
\end{abstract}


this period, was also noticed. The most productive institutions are the Universidade Estadual Paulista - UNESP and the Federal University of Minas Gerais - UFMG, institutions that had at least one author affiliated in half of the institutional implications. There is also a strong growth in the number of articles in the most recent years, especially in 2012 and 2014 onwards, responsible for almost $85 \%$ of the total number of Brazilian publications in the journal. Relative to national coauthories, a strong group was noticed worried about the ethical and social questions in knowledge organization, studies in documentary languages, aspects relative to information retrieval, semantic web, ontologies, beside others. Regarding international co-authoring, emphasis is placed on thematic interest groups, such as ethical and social dimensions, bibliometric aspects, interlocution with the exact sciences and technologies, among others. Conclusions: The leadership of the group of authors confirms that the journal is open on topics such as ethical and social aspects of $\mathrm{OC}$, as well as technological perspectives in the context of this domain. In addition, a strong incidence of affiliated articles between UNESP and UFMG has revealed a specificity and a research tradition of these two universities on which knowledge organization is based.

Descriptors: Domain Analysis. Epistemic Communities. Brazilian scientific production. Knowledge Organization.

\title{
LA PRESENCIA DE LA PRODUCCIÓN CIENTÍFICA BRASILEÑA EN LA REVISTA KNOWLEDGE ORGANIZATION EN EL SIGLO XXI
}

\begin{abstract}
RESUMEN
Introducción: La revista Knowledge Organization es hoy uno de los principales vehículos para la comunicación científica con respecto a los problemas de la Organización del Conocimiento (CO). Objetivo: Por lo tanto, se consideró pertinente analizar cómo se presenta el contexto brasileño en estas comunicaciones, verificando cuáles son los autores e instituciones más productivos, así como la frecuencia de las publicaciones, la coautoría nacional y las colaboraciones con el extranjero. Metodología: Através del análisis de los números publicados en el período 20012018, fue posible identificar una fuerte presencia de artículos de autores brasileños, con un total de 51 producciones. Resultados: Una comunidad dirigida por los maestros José Augusto Chaves Guimarães, Daniel Martínez-Ávila, Fabio de Assis Pinho y Renato Rocha de Souza, que son responsables del $28 \%$ de todas las publicaciones en este período. Como instituciones más productivas, destacamos la Universidad Estatal Paulista - UNESP y la Universidad Federal de Minas Gerais UFMG, que tenía al menos un autor afiliado en la mitad de las incidencias institucionales. También hay un fuerte crecimiento en el número de artículos en los últimos años, especialmente en 2012 y 2014 en adelante, lo que representa casi el $85 \%$ de todas las publicaciones brasileñas en la revista. Con respecto a la coautoría nacional, podemos ver un grupo fuerte preocupado por los aspectos éticos y sociales de la organización del conocimiento, estudios en lenguajes documentales, aspectos relacionados con la recuperación de información, ontologías, web semántica, entre otros. Con respecto a la coautoría internacional, se destacan los grupos de interés temáticos, como las dimensiones éticas y sociales, los aspectos bibliométricos, el diálogo con las ciencias y tecnologías exactas, entre otros. Conclusiones: E
\end{abstract}

Inf. Inf., Londrina, v. 24, n. 3, p. 28 - 51, set./dez. 2019. 
liderazgo del grupo de autores confirma que la revista está abierta sobre temas como los aspectos éticos y sociales de OC, así como las perspectivas tecnológicas en el contexto de este dominio. Además, una fuerte incidencia de artículos afiliados entre UNESP y UFMG ha revelado una especificidad y una tradición de investigación de estas dos universidades en las que se basa la organización del conocimiento

Descriptores: Análisis de domínio. Comunidades epistémicas. Producción científica brasileña. Knowledge Organization. 


\section{APÊNDICE A - REDE DE COAUTORIA NACIONAL}

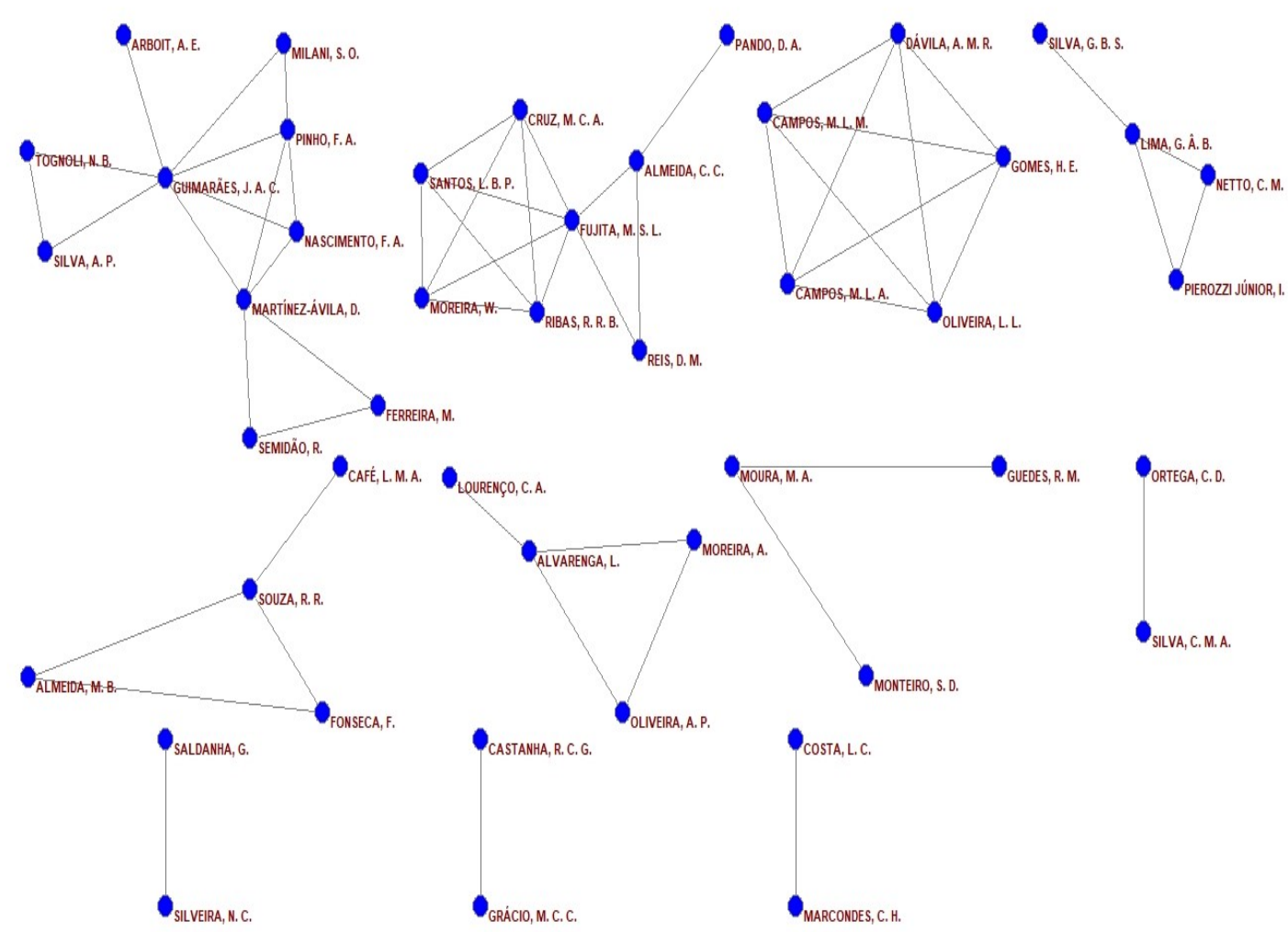




\section{APÊNDICE B - REDE DE COAUTORIA INTERNACIONAL}

Legenda: AMARELO = pesquisadores internacionais; AZUL = pesquisadores nacionais.
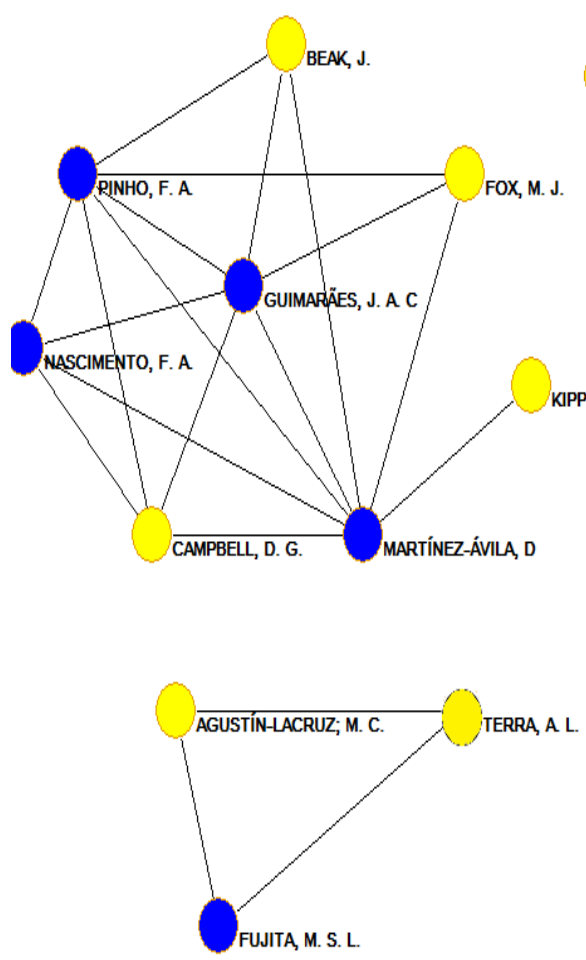

FUJITA, M. S. L.

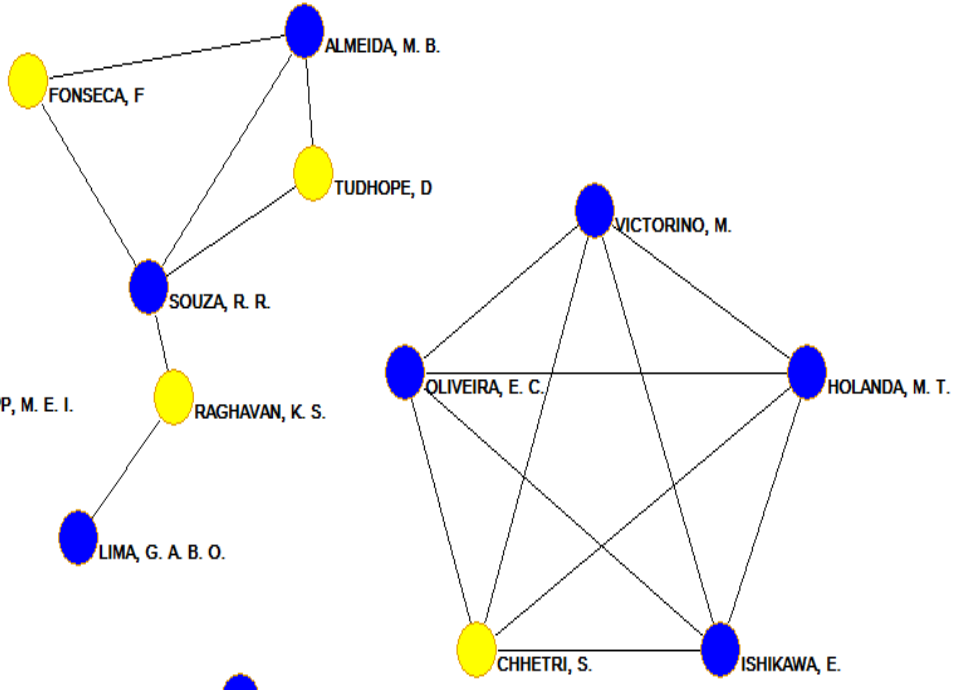

CAstanha, R. C. G

WOLFRAM, D. 